\title{
Detecting and Analyzing the Malicious Social Bots by using Data Mining and Naïve Bayesian Classifier
}

\author{
M Vinay Sai ${ }^{1}$, Gandharba Swain ${ }^{2}$, K Hari Kishore ${ }^{3}$ \\ ${ }^{1}$ M.Tech Student, Department of CSE, Koneru Lakshmaiah Education Foundation, Vaddeswaram, Guntur, A.P, \\ India, vinaysaimuppalla@gmail.com \\ ${ }^{2}$ Professor, Department of CSE, Koneru Lakshmaiah Education Foundation, Vaddeswaram, Guntur, A.P, India \\ ${ }^{3}$ Professor, Department of ECE, Koneru Lakshmaiah Education Foundation, Vaddeswaram, Guntur, A.P, India
}

\begin{abstract}
With the huge increase in the number, speed, and variety of customer information (such as customer-generated information) in online interpersonal organizations, people have worked hard to construct better methods to collect and inspect such large information. For example, social robots have been used to perform scientific management of robots and provide customers with improved management attributes. Even so, harmful social robots are used to spread false data (for example, false news), which may bring real results. Therefore, identifying and evacuating harmful social robots in online informal communities is crucial. The latest discovery technology of resentful social robots undermines the quantitative focus of its behavior. These highlights are easily imitated by social robots. This leads to a reduction in the accuracy of the investigation. This article describes an epic strategy for identifying malicious social robots, which includes two key options, depending on the possibility of snapshot stream grouping and semi-hosted binding changes. The technology not only investigates the possibility of changes in the click stream of customer behavior, but also takes into account the temporal highlights of the behavior.
\end{abstract}

Key words: Online social network, social robots, Customer behavior, semi-supervised clustering.

\section{INTRODUCTION}

Data Mining is a method that organizations use to turn raw data into usable information. Using algorithms to scan trends in vast volumes of data, corporations can learn more about their clients and create more successful content campaigns, boost revenue and lower expenses. Data mining relies on effective data collection, storage, and computer processing. Supermarkets are well-known customers of information mining methods. Many grocery stores provide customers with free membership cards, so that they can get preferential prices that non-individuals cannot enjoy. Such cards make it easier for shops to keep track of who ordered what at what price and when. Once the information has been disaggregated, the shop would be able to utilize this information and provide consumers with coupons customized and their purchase preferences, and chose whether to reduce the products mentioned or market them to the maximum degree practicable. When an organization uses only selected data (it cannot explain a general set of examples) to illustrate a particular theory, information mining may be the cause of concern. Data mining is a method of analysis designed to analyze data (typically massive volumes of data, generally relevant to industry or market, also known as 'big data') to locate common patterns and/or machine relationships between variables, and then to validate the findings by extending the trend found to a new subset of data. A definite aim of information mining is prescient, and prescient information mining is the most commonly accepted method of information mining and the most immediate application for industry. The knowledge mining technique includes three phases: (1) the primary inquiry (2) the model layout or sample validation with clarification/check, and (3) the arrangement.

\section{LITERATURE SURVEY}

Some of the issues commonly associated with anonymity online are that it impedes a sense of social obligation, as has been demonstrated by a great deal of fake news online. Despite the lack of prompts for identification in cyberspace, people still leave fragments of textual identities behind. In this report, we recommend using methods for the stroke examination to better classify individuals based on writing style. They incorporate a rich range of features of the type, including terminology, grammar and form, content-specific attributes and functionality [1], [5]. We have also developed Write prints technology for anonymous identification and similarity detection. Write prints is a technology based on the Karhunen-Loeve transform, which uses sliding windows and mode interrupt algorithms in combination with a separate author-level feature set. On a test bench containing four online datasets spanning different fields, the Write prints technology and extended feature set were evaluated: email, instant messaging, feedback comments, and program code.

\section{EXISTING METHODOLOGIES}

We are improving the current architecture for a Scalable and Robost Truth Discovery (SRTD) scheme to tackle the above three challenges. In fact, using a rational approach, the SRTD scheme mutually quantifies both the authenticity of data and the credibility of statements. Using Function Queue in a 
Condor environment we further establish a centralized mechanism for applying the suggested truth discovery scheme.

The major works of this study are:

1) Collected from unstructured fictitious information: In the field of fake news evaluation, most evaluations use the formed information for information disclosure. In this figure, we consider mining access logs to perceive important data from key data. The conversation log contains some social occasions written in conventional languages. Conference discussions are always short and often cause confusion and language confusion. So try to determine the data extraction [6].

2) Customized definition of networks mined by artificial communities: standard techniques of network analysis typically calculate the amount of direct connections between users, e.g. the number of emails exchanged. Having consulted with law enforcement officials, we find that it is not possible to consider just the amount of direct contacts, and can result in knowledge loss or inconsistent outcomes. Therefore a personalized term for groups that mine fake communities is described in this report. In the sense of chat history mining, even though there is no direct interaction, the individuals which always appear in the chat conversation are known as a group [7].

3) Concept recognition without any previous knowledge: many of the latest concept recognition techniques allow researchers to provide training data to test classification models. Nevertheless, it is not straightforward to collect this evidence in the case of fake news inquiries. Our suggested structure does not include any background knowledge or preparation data and will define core concepts (or topics) depending on the nature of the conversation with the aid of the WorldNet vocabulary database [8]. In comparison, the term extraction method depends on the conceptual similarity of terms rather than on the frequency of terms [9]. Our technology is also in a role to determine the most important terms that can best reflect and interpret the knowledge in the chat session.

4) Flexible usage of domain information: our proposed compromised collective mining platform enables researchers to incorporate domain knowledge in order to enhance the study method. Domain awareness may be the term taxonomy used in deceptive online communications, and it reflects a street language with fake news.

The overview of SRTD system is shown in Figure 1.Also its dynamic feedback system is shown in Figure 2.



Figure 1: SRTD System Overview



Figure 2: Dynamic Feedback Control System

\section{PROPOSED METHODOLOGY}

News falsification is one of the most important issues for our listeners, and its expectations are high. Committed to. There will be a lot of false news for most of the day [2]. This requires tracking all false news and keeping the database for future reference [10], [12]. The challenge now is to maintain a set of legitimate false news and disseminate this information to help predict and understand false news in the future.

The purpose of this project is to analyze a data set composed of a large number of fake news and predict the types of fake news that may occur in the future according to various circumstances. In this article, we propose a method of using data mining for fake news prediction and classification. Here, we use a simple Bayesian classifier for fake news prediction and classification.

Knowledge is a fraudulent defendant whose identities are specifically linked to an event or are loosely connected to fakes. Due to the principle of confidentiality, obtaining false news datasets in practice is a difficult process. Therefore, fake news datasets are synthesized using the latest methods. At Naive Bayes, the technology was tested. Verification and cross-validation shall be used to verify the performance of growing systems. Experimental tests demonstrate that we can achieve better classification precision by using cross-validated Nive Bayes.

\subsection{Naïve Bayes Classifier Model}

In this section, the proposed naive Bayesian network model is designed to resolve the fabricated question of prediction. Naive Bayes Classifier is a basic probabilistic classifier that operates by implementing Bayer's theorem and naive assumptions about the freedom of the components. Despite the presumption of freedom, the basic Bayesian classifier has proven to be very useful in modeling the conditions of complex practical problems. Throughout this segment, the incident variable is displayed throughout bold and lowercase fonts, while the meaning of the incident variable is shown in the uppercase Italian font. The system model for fake prediction is shown in Figure 3. 
The proposed model is constructed to represent the date and location of the event, fake news and pseudonyms, and the acquaintance of each dummy as clues. When used as a learner, the better model is the one that maximizes learning efficiency, not the one that better reflects the true relationship between variables. In this case, the fake news, geographic location and date variables are considered valid for the fake variables. The network of fake acquaintances also has an impact on dummies. But here, acquaintances other than acquaintances (second acquaintances) have negligible influence on the results and reduce the computational complexity, so these acquaintances are not considered.

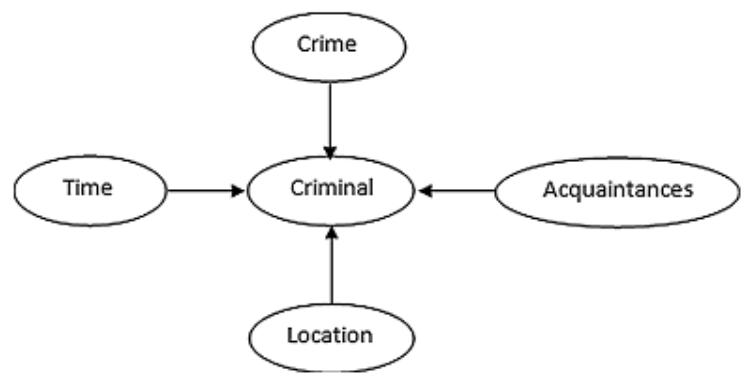

Figure 3: System model for fake prediction

The proposed model shown in Figure 4 is constructed to represent the date and location of the event, fake news and pseudonyms, and the acquaintance of each dummy as clues. When used as a learner, the optimal model is one that maximizes learning efficiency, not the one that better represents the true relationship between variables [11].

In this scenario, false news, geographical position and date variables are known to be true for inaccurate variables. The network of fake acquaintances also has an impact on dummies. But here, acquaintances other than acquaintances (second acquaintances) have negligible influence on the results and reduce the computational complexity, so these acquaintances are not considered.

The proposed model is constructed to represent the date and location of the event, fake news and pseudonyms, and the acquaintance of each dummy as clues. When used as a learner, the better model is the one that maximizes learning efficiency, not the one that better reflects the true relationship between variables.

In this case, the fake news, geographic location and date variables are considered valid for the fake variables [3], [4]. The network of fake acquaintances also has an impact on dummies. But here, acquaintances other than acquaintances (second acquaintances) have negligible influence on the results and reduce the computational complexity, so these acquaintances are not considered.

The second component is multinomial, which applies the naïve Bayes algorithm for distributed multinomial results, usually used for discrete counts. The third method is Gaussian, where the frequency of the features is considered to be Gaussian, and we have constant features instead of distinct counts. Regression and classification features were used in the Scikit-learn database.



Figure 4: Proposed model for fake prediction

\section{RESULTS}

The results of the proposed method are depicted in the figures 5-19.

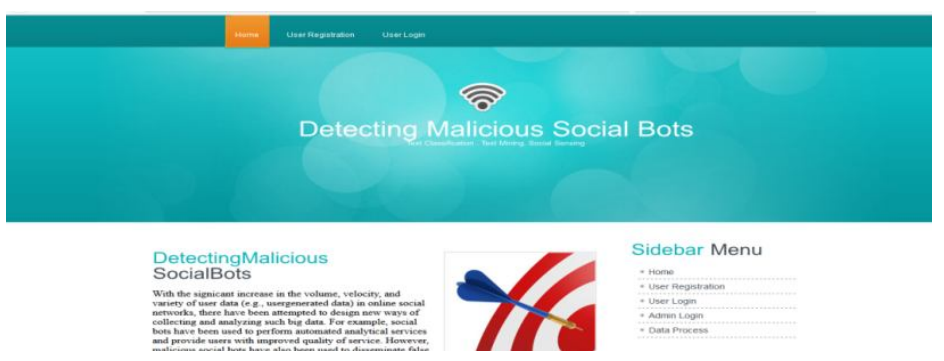

Figure 5: Home page in the detection of Malicious Social Bots



Sidebar Menu

- Home

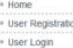

Registration success page

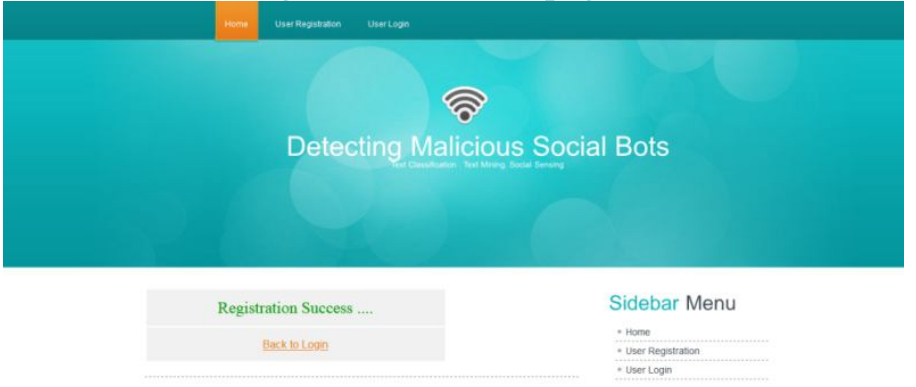

Figure 7: Registration Success page in the detection of Malicious Social Bots 
M Vinay Sai et al., International Journal of Emerging Trends in Engineering Research, 8(7), July 2020, 3345 - 3350

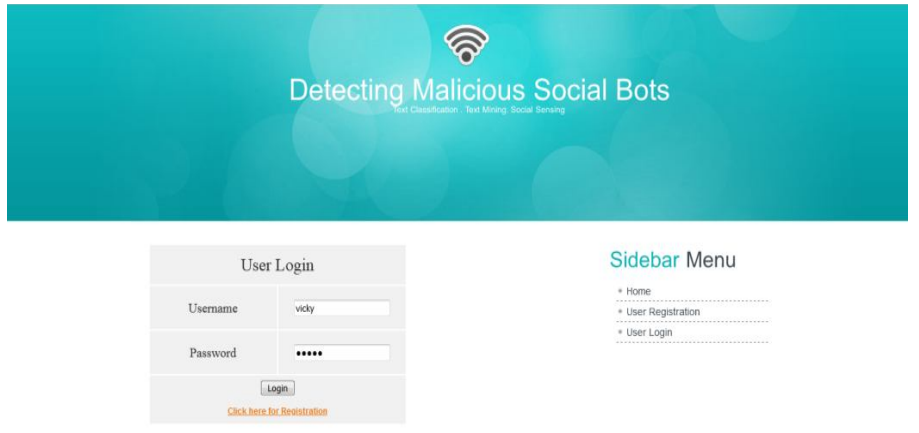

Figure 8: User Login page in the detection of Malicious Social Bots
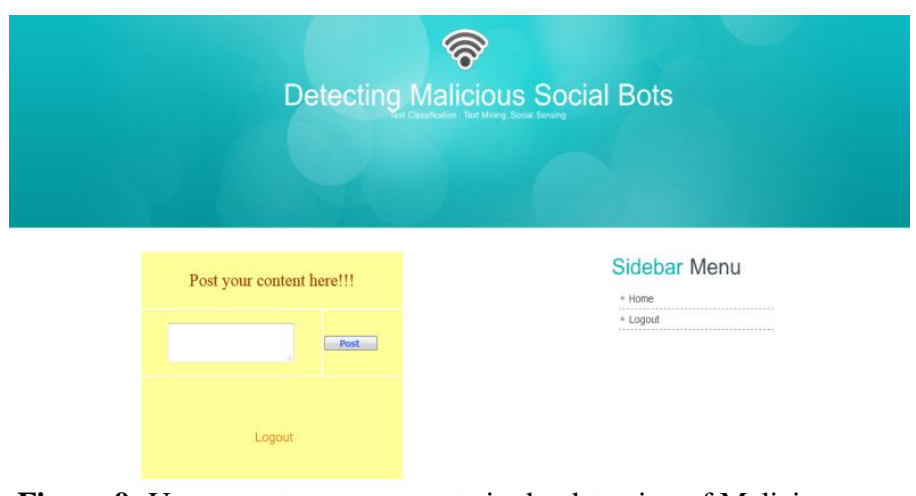

Figure 9: User page to post contents in the detection of Malicious Social Bots


Sidebar Menu

- Home

Figure 10: Posting Normal contents in the detection of Malicious Social Bots

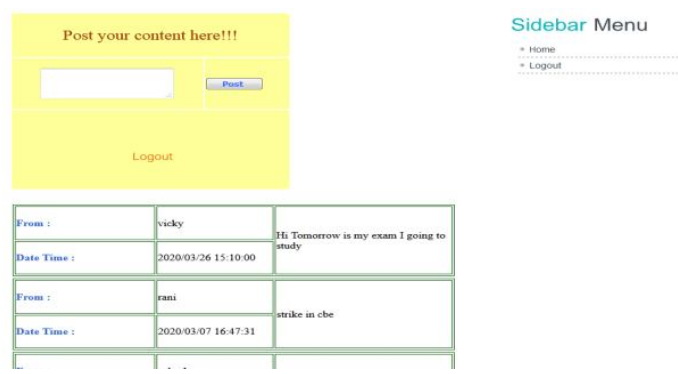

Figure 11: Normal post displayed in the detection of Malicious Social Bots
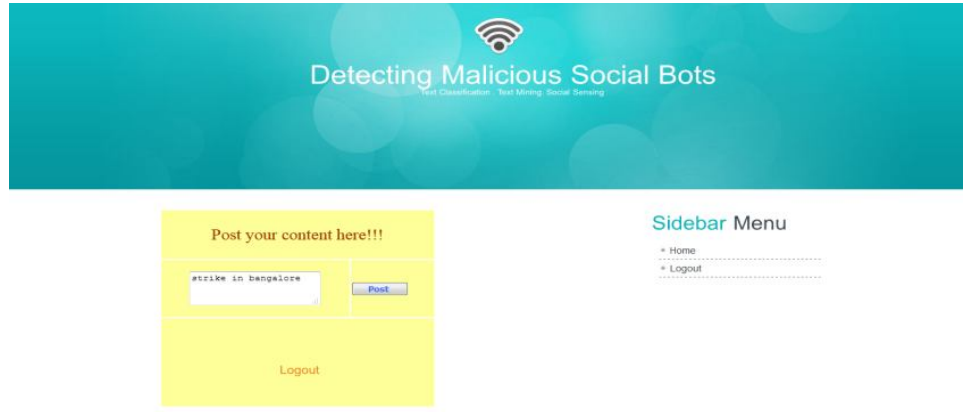

Sidebar Menu



Figure 12: Fake news post in the detection of Malicious Social Bots



Figure 13: Fake news viewed by only Admin, if fake news is posted(Admin login page) in the detection of Malicious Social Bots

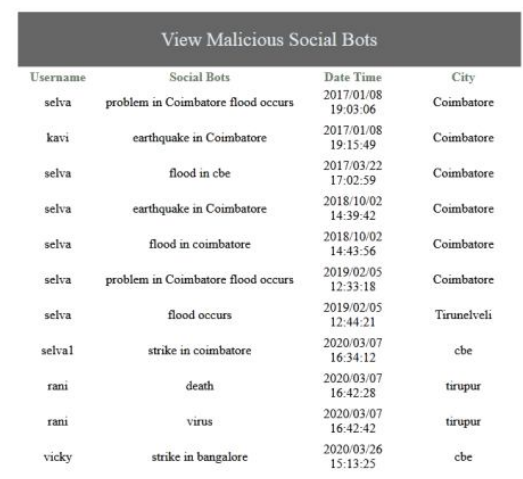

Figure 14: Admin view for fake news post in the detection of Malicious Social Bots

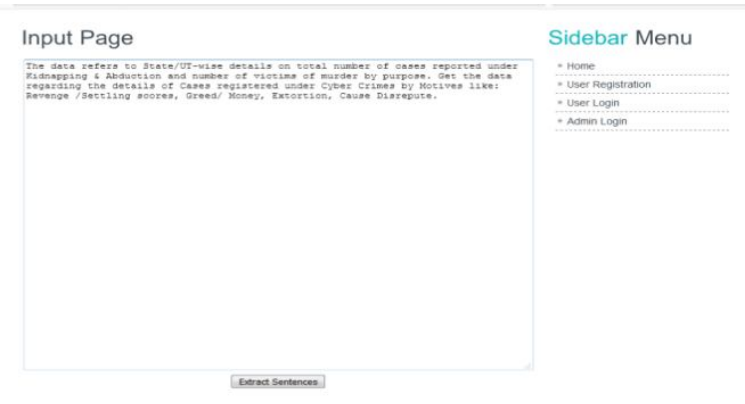

Figure 15: Background data processing in the detection of Malicious Social Bots 


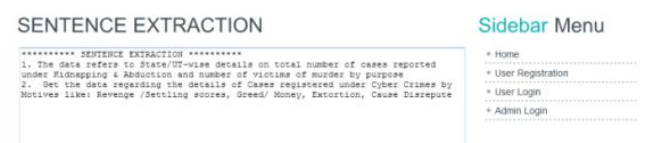

Figure 16: Sentence extraction- split paragraph into sentence in the detection of Malicious Social Bots

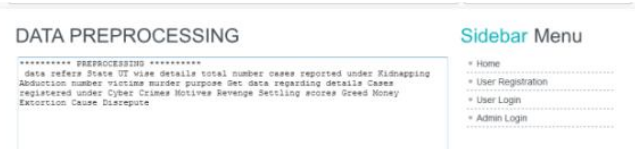

Figure 17: Pre-processing- Removing the stop words in the detection of Malicious Social Bots

\section{Extracting the terms}

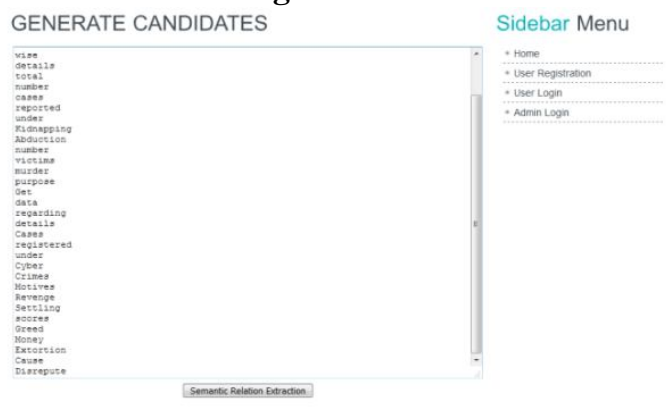

Figure 18: Extracting the terms in the detection of Malicious Social Bots

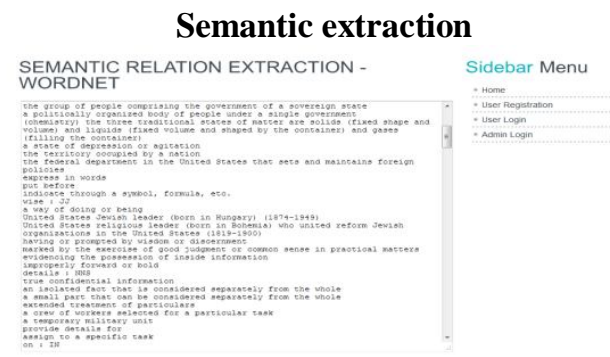

Figure 19: Semantic Extraction in the detection of Malicious Social Bots

\section{CONCLUSION}

A functional model is introduced, based on the naive Bayes classifier, and a new principle is added to the question of false prediction. The fake news knowledge at plot level is generated automatically by the paradigm itself, so it's hard to accomplish in reality anyway. The suggested model is functional, owing to the simplicity of Naive Bayes' assertion of equality. The model is indeed quite good for autonomy questions, as the model has accomplished the job of raising the number of speculators by $80 \%$. Study reports suggest that the paradigm can be applied with an overall performance rate of 78.05 percent in criminology, and will allow intelligence forces to assess the nature of the accident. The new model's radical critique is its capacity to include workers in complex processes. This research facilitates further analysis of voice demand problems through its latest era- based complex speech news dataset framework.

\section{REFERENCES}

[1]. C. A. De Lima Salge and N. Berente, Is that social bot behaving unethically?, Commun. ACM, vol. 60, no. 9, pp. 29-31, Sep. 2017. https://doi.org/10.1145/3126492

[2]. M. Sahlabadi, R. C. Muniyandi, and Z. Shukur, Detecting abnormal behavior in social network websites by using a process mining technique, J. Comput. Sci., vol. 10, no. 3, pp. 393-402, 2014. https://doi.org/10.3844/jcssp.2014.393.402

[3]. F. Brito, I. Petiz, P. Salvador, A. Nogueira, and E. Rocha, Detecting Social-Network Bots Based On Multiscale Behavioral Analysis, in SECURWARE 7th Int. Conf. Emerg. Secur. Inf., Syst. Technol., Barcelona, Spain, 2013, pp. 81-85.

[4]. T. K. Huang, M. S. Rahman, H. V. Madhyastha, M. Faloutsos, and B. Ribeiro, An Analysis Of Socware Cascades In Online Social Networks, in Proc. 22nd Int. Conf. World Wide Web, Rio de Janeiro, Brazil, 2013, pp. 619-630. https://doi.org/10.1145/2488388.2488443

[5]. H. Gao, Y. Yang, K. Bu, Y. Chen, D. Downey, K. Lee, and A. Chowdary, Spam ain't as diverse as it seems: throttling OSN spam with templates underneath, in Proc. 30th ACSAC, New Orleans, LA, USA, 2014, pp. 76-85. https://doi.org/10.1145/2664243.2664251

[6]. E. Ferrara, O. Varol, C. Davis, F. Menczer, and A. Flammini, The rise of social bots, Commun. ACM, vol. 59, no. 7, pp. 96-104, Jul. 2016. https://doi.org/10.1145/2818717

[7]. T. Hwang, I. Pearce, and M. Nanis, Social Bots: Voices From The Fronts, Interactions, vol. 19, no. 2, pp. 38-45, Mar. 2012. https://doi.org/10.1145/2090150.2090161

[8]. Y. Zhou, D. W. Kim, J. Zhang, L. Liu, H. Jin, H. Jin, and T. Liu, Detecting Malicious Accounts In Social-Network-Based Online Promotions, IEEE Access, vol. 5, pp. 1990-1999, 2017. https://doi.org/10.1109/ACCESS.2017.2654272

[9]. Z. Zhang, C. Li, B. B. Gupta, and D. Niu, Efficient compressed cipher text length scheme using multi-authority cp-abe for hierarchical attributes, IEEE Access, 2018. 
[10]. Chella Santhosh, K. Hari Kishore, G. Pavani Lakshmi, G.Kushwanth, P. Rama Krishna Dharma Teja, R. S. Ernest Ravindran, Sree Vardhan Cheerala, M. Ravi Kumar "Detection of Heavy Metal Ions using Star-Shaped Microfluidic Channel" International Journal of Emerging Trends in Engineering Research, ISSN: 2347-3983, Volume-7 Issue-12, Page No: 768-771, December 2019. https://doi.org/10.30534/ijeter/2019/067122019

[11]. B. Srikanth, M. Siva Kumar, J.V.R. Ravindra, K. Hari Kishore "Double Precession Floating Point Multiplier using Schonhage-Strassen Algorithm used for FPGA Accelerator" International Journal of Emerging Trends in Engineering Research, ISSN: 2347-3983, Volume-7 Issue-11, Page No: 677-684, December 2019.

https://doi.org/10.30534/ijeter/2019/437112019

[12]. Radhika Rani Chintala, Lakshmi Sri Ram Janjanam, Sai Kousik G, Sai Pawan S 'FPGA Implementation of Katan Block Cipher for Security in Wireless Sensor Networks" International Journal of Emerging Trends in Engineering Research , ISSN: 2347-3983, Volume-7 Issue-11 Page No: 492-497, December 2019.

https://doi.org/10.30534/ijeter/2019/157112019 